\title{
ISOMORPHISM INVARIANCE AND OVERGENERATION
}

\author{
OWEN GRIFFITHS AND A.C. PASEAU
}

\begin{abstract}
The isomorphism invariance criterion of logical nature has much to commend it. It can be philosophically motivated by the thought that logic is distinctively general or topic neutral. It is capable of precise set-theoretic formulation. And it delivers an extension of 'logical constant' which respects the intuitively clear cases. Despite its attractions, the criterion has recently come under attack. Critics such as Feferman, MacFarlane and Bonnay argue that the criterion overgenerates by incorrectly judging mathematical notions as logical. We consider five possible precisifications of the overgeneration argument and find them all unconvincing.
\end{abstract}

The standard approach to logical consequence in the modern literature is the post-Tarskian model-theoretic conception. On this conception, a sentence $\phi$ is a logical consequence of some set of sentences $\Gamma$ just if every model of $\Gamma$ is a model of $\phi$. A model consists of a nonempty domain of objects and a valuation function that assigns semantic values to the nonlogical expressions of the language. As such, the standard approach relies on a distinction between logical and nonlogical expressions. It is striking, then, that there is little agreement over how this crucial distinction should be drawn. We defend the most natural supplement to the model-theoretic definition: isomorphism invariance.

The isomorphism invariance criterion of logical nature has much to commend it. It can be philosophically motivated by the thoughts that logic is distinctively general or topic-neutral. It is capable of precise set-theoretic formulation. And it delivers an extension of 'logical constant' which respects the intuitively clear cases, namely identity, the Boolean connectives and the universal and existential quantifiers - the logical constants of first-order logic with identity.

Despite its attractions, isomorphism invariance faces a major recurring objection in the literature. Critics such as Christopher Peacocke [20], Timothy McCarthy [15], Solomon Feferman ([8, 9, 11]), John MacFarlane [13], Enrique Casanovas [5], Denis Bonnay ([1, 2]), and Catarina Dutilh Novaes [6] all argue that the criterion overgenerates by incorrectly judging some mathematical notions as logical. Though he does not discuss isomorphism invariance, the same criticism is implicit in Tharp [31].

Received April 13, 2016.

2010 Mathematics Subject Classification. 03A05, 03B10, 03B15, 03 C98.

Key words and phrases. isomorphism invariance, logical constants, second-order logic, logical consequence, model theory, overgeneration argument. 
Our paper has three objectives. First, we defend isomorphism invariance as an attractive account of logical nature. We believe that the isomorphism invariance approach can survive this major objection. We consider several possible precisifications of the objection and find them all unconvincing. Second, much of our discussion is available to the defender of second-order quantification as genuinely logical. Third, our discussion will help clarify the demarcation of logic from mathematics.

\section{§1. The isomorphism-invariance account.}

1.1. Brief motivation. A popular slogan is that logic is formal. Quite what the slogan amounts to is a controversial matter but even a minimal reading is committed to valid arguments having purely valid forms: argument forms all of whose instances are truth-preserving. ${ }^{1}$ For example, the propositional argument $(P \vee Q) \wedge R \therefore P \vee Q$ has a purely valid form expressed by $\Phi_{1} \wedge \Phi_{2} \therefore \Phi_{1}$ (provided we replace the schematic variables $\Phi_{1}$ and $\Phi_{2}$ uniformly).

In his famous paper on logical consequence, Tarski accepts this much but notes that the characterisation of formality is incomplete because ' $[\mathrm{u}]$ nderlying the whole construction is the division of the language into logical and extralogical' ([28], p. 418). Such formal accounts of logical consequence must be supplemented with a demarcation of the logical constants.

Tarski thought that the logical constants are:

terms of a much more general character occurring in most statements of arithmetic, terms which are met constantly both in considerations of everyday life and in every field of science. ([29], p. 18)

The purpose of logic, he continues, is to 'establish the precise meaning of such terms' and to 'lay down the most general laws in which these terms are involved' ([29], p. 18).

Tarski's thought is that the logical constants are those expressions, such as 'not', 'and', and 'every', that are required to carry out inference in any field whatsoever and which feature in the most general laws. They are not expressions that have any particular subject matter themselves, but are rather required in all disciplines if we are to reason correctly. In ([30]), he put forward an invariance criterion as a precise test for demarcating the logical constants, understood as the most general pieces of vocabulary. In this paper, he defines logic as the 'science which deals with the notions invariant under the widest class of transformations', which will be 'very few notions, all of a very general character' ([30], p. 149).

This motivation for isomorphism invariance stems from the thought that logic is distinctively general. We could instead motivate the account with the thought that logic is distinctively topic-neutral. ${ }^{2}$ If logic is topic-neutral, then the logical operations should be insensitive to the particular objects to which

\footnotetext{
${ }^{1}$ See Oliver [18] for a good discussion of the slogan.

${ }^{2}$ See ([1], pp. 33-35) for more on the distinction between the two sorts of motivation.
} 
they are being applied. In particular, they should be insensitive to arbitrary switching of objects. ${ }^{3}$ If a notion is invariant under isomorphism, then there is a clear sense in which it is insensitive to the arbitrary switchings of objects. The topic-neutrality of logic, explicated in terms of sensitivity to the switching of objects, therefore offers a distinct motivation for isomorphism invariance. ${ }^{4}$ In this paper, we remain neutral between these two means of motivating isomorphism invariance.

1.2. Brief statement. Invariance criteria for logical nature first define isomorphism invariance for the worldly entities (objects, sets, ordered $n$-tuples, etc) that are the extensions of linguistic expressions, and then derivatively for those expressions. An expression is then invariant in the appropriate respect just if its extension is invariant.

The expressions that we usually take to be candidate logical constants, however, are connectives and quantifiers, which do not have extensions in a straightforward sense. Along with the majority of writers in this area, we follow McGee ([16], p. 569) in taking the extension of an $n$-ary quantifier or connective $C$ on domain $D$ as a function from an $n$-tuple of sets of variable assignments of values from $D$ to a set of such assignments. Intuitively, if the input of such a function is the $n$-tuple of sets of assignments that satisfy $\phi_{1}, \ldots, \phi_{n}$, then the output is the set of assignments that satisfy $C\left(\phi_{1}, \ldots, \phi_{n}\right) .{ }^{5} \mathrm{McGee}$ goes on to prove that the invariant expressions are exactly those that are definable in the language $\mathcal{L}_{\infty, \infty}$. Although a more formal account of all these ideas could be given, our arguments in this paper do not require anything more than the informal characterisation just presented.

The Tarski-Sher Thesis states that an expression of type level at most 2 is a logical constant just if its semantic value is isomorphism invariant. This type restriction is in place to limit attention to first-order languages but, if topicneutrality is the motivation, then we find no reason for such a restriction. As such, the thesis we defend is simply that an expression is a logical constant just if its semantic value is isomorphism invariant.

1.3. Objections. Before considering the overgeneration charge in detail, we will briefly map out the objections that have been raised to isomorphism invariance. Feferman ([9], p. 6) summarises the standard objections as follows:

1. The thesis assimilates logic to mathematics, more specifically to set theory.

2. The set-theoretical notions involved in explaining the semantics of $\mathcal{L}_{\infty, \infty}$ are not robust.

\footnotetext{
${ }^{3}$ See ([13], Section 3.2) for more on this understanding of topic-neutrality.

${ }^{4}$ Sher ([26], pp. 192-194) motivates isomorphism invariance by its ability to explain the formality, generality and topic-neutrality of logic. She also holds that it can explain logic's strong modal force, its normativity and its a priori character.

${ }^{5}$ In line with the literature we are concerned with, we take logical constants to be defined over models. Though as Sher ([25], p. 56) points out, a natural invariance condition implies that a logical term will have the same extension in any two models that share a domain.
} 
3. No natural explanation is given by it of what constitutes the same logical operation over arbitrary basic domains.

Our central concern is the first objection. The second charge is that the set-theoretical notions involved in the isomorphism invariance account are not robust. ${ }^{6}$ The worry seems to be that, although isomorphism of structures is defined in a structural, language-independent, way (as opposed to e.g., elementary equivalence), the existence of an isomorphism between any two structures depends on the ambient universe of sets. To put it in familiar set-theoretic terms, the relation of isomorphism between models is not absolute. $^{7}$

Feferman writes that the absoluteness objection 'is in a way subsidiary to [the overgeneration objection]' ([9], p. 9). An obvious interpretation is that the absoluteness objection is 'subsidiary' in the sense of being of secondary importance. He admits that the absoluteness objection is 'vague' ([9], p. 9) but the overgeneration problem it gives rise to is capable of precise expression. We choose to focus on the overgeneration argument in part because it is clearer than the subsidiary absoluteness problem. A further reason is that neither Feferman nor Bonnay motivates the thought that the set-theoretic notions involved in isomorphism invariance should be robust. One obvious motivation would be anti-realism about set theory, since a realist about sets would maintain that the question of whether two structures are isomorphic has a definite answer. ${ }^{8}$ Another motivation could be an independence requirement: the analysis of logicality should be independent of mathematics, and set theory in particular. We reject anti-realism about sets, and hold that the independence motivation sits poorly with isomorphism invariance, since it is designed to supplement the model-theoretic definition of logical consequence, which is set-theoretic through-andthrough. Of course, these are not knock-down objections, but reasons to focus on overgeneration. ${ }^{9}$

Another famous problem for the account, not listed here by Feferman, is that the isomorphism-invariance account ignores the meanings of logical constants. Anything co-extensive with a logical constant is a logical constant, such as McGee's ([16], p. 569) famous example of unicorn-negation:

$$
\mathcal{U} \phi=_{\text {Def }}(\neg \phi \wedge \text { there are no unicorns }) \text {. }
$$

A related example of a connective that operates in the same way on domains of the same size would be a connective that acts (i) as disjunction when the domain is an even successor cardinal, (ii) like conjunction when the domain

\footnotetext{
${ }^{6}$ Bonnay ([1], p. 60) also raises this objection.

${ }^{7}$ To take a simple example, consider a structure $\mathfrak{A}$ with domain $\alpha$ for $\alpha$ some countably infinite ordinal $(\neq \omega)$, and a structure $\mathfrak{B}$ with domain the least infinite ordinal $\omega$. In some countable transitive model $M$ of $\mathrm{ZFC}, \mathfrak{A}$ and $\mathfrak{B}$ may not be isomorphic, for example if $M$ does not contain a bijection from $\alpha$ to $\omega$ (so that $\alpha$ is uncountable in $M$ ), although $\mathfrak{A}$ may be isomorphic to $\mathfrak{B}$ in some generic extension of $M$ (that satisfies the axioms of ZFC).

${ }^{8} \mathrm{By}$ set realism, we understand the thesis that there is a single definite universe of sets and that ZFC, as usually interpreted, is true of this universe. Anti-realism is then any view opposed to realism. Some set theorists (e.g., [12], p. 416) call set realism 'the universe view'.

${ }^{9}$ See Sher ([27], pp. 307-311) for another response.
} 
is an odd successor cardinal, and (iii) like a biconditional at limit cardinals ([16], p. 577). This objection was first raised by McCarthy [15]. At the sentence level, the worry is that, if unicorn-negation is a logical constant, then a sentence such as

$$
U(0 \neq 0 \vee \mathcal{U} 0 \neq 0)
$$

is a logical truth, which is implausible.

Again, however, the overgeneration argument is more basic. The overgeneration argument - if successful - is devastating for proponents of isomorphism invariance. They sought a demarcation of the logical constants to supplement the standard definition of logical consequence; if they have to call palpably nonlogical sentences logical truths, then they have failed at their task. In contrast, faced with cases such as unicorn-negation, it is open to the proponent of isomorphism invariance to emphasise the extensional nature of their project; unicorn-negation, after all, is coextensional with negation tout court. Gil Sagi [22] has recently taken this line: her strategy is to bite the bullet and defend the logical truth of $U$. One reason to deny the logical truth of $U$ is its apparent contingency. ${ }^{10}$ But, she argues, if we hold that meaning is determined by extension, and that we must hold meaning fixed when evaluating the truth-value of a sentence at different worlds, then $U$ is true at every world. In a world with unicorns, we may be tempted to say that $U$ is false, but that is to change the meaning of ' $\mathcal{U}$ ': when meaning is held fixed, the sentence is necessarily true. A different idea than Sagi's that has been discussed ([13]) and could be exploited in this connection is that logical constants ought to be lexically primitive, a test presumably failed by unicorn-negation.

Finally, isomorphism invariance could be criticised for undergenerating. Criticisms of this sort are much harder to find in the literature. As far as we are aware, the only writers who discuss the undergeneration of isomorphism invariance are Dutilh Novaes [6], ${ }^{11}$ Woods [32] and MacFarlane [14]. We will focus on the far more developed overgeneration problem.

Our discussion therefore focusses on the major objection to arguably the most plausible and best-developed account of logical constanthood. Our second aim is to defend second-order quantification as logical from an analogous objection. Our discussion's third moral, which will emerge as we go along, is that the boundary between mathematics and logic is firmer than is often supposed.

Though isomorphism invariance is the best-known invariance account, the problems outlined have motivated Bonnay and Feferman to offer distinct invariantist accounts. Although we do not think that these are as well-motivated as the isomorphism account, we content ourselves in this

\footnotetext{
${ }^{10}$ Sagi also considers the objection that $U$ is not a logical truth because it is a posteriori.

${ }^{11}$ Dutilh Novaes charges isomorphism invariance with undergeneration by failing to find the S4 logical operators to be logical. But if you accept - as Burgess [4] argues convincingly that we should - that the correct modal logic for logical necessity is S5, then you will be untroubled by Dutilh Novaes' arguments, which do not threaten the logical status of the S5 operators, at least when interpreted on universal frames.
} 
article to defending isomorphism invariance and do not consider Bonnay and Feferman's respective positive proposals. ${ }^{12}$ Bonnay writes that

In the setting of generalized invariance, it does not make sense to require full generality without qualification, because the most general notions are as much useless as they are general.... Logical notions are the most general notions which deal with certain kinds of features; but in order to make sense of the generality of logic, one has to say what matters to logic. ([1], pp. 48-49)

We agree: there is a limit to how general we can go here, since some transformations will yield criteria that clash wildly with intuition and practice. So to deliver an acceptable criterion we cannot blindly follow generality to the very limit, but instead need to settle "what matters to logic'. As it is beyond the scope of our paper to do that, all we'll note here is that one major motivation for generalising beyond Tarski-Sher is the overgeneration problem, which is what we aim to defeat in this paper. Other problems - such as intensional worries or undergeneration worriesapply equally to all criteria. The robustness problem is directly targeted at isomorphism invariance but, as we have noted, Feferman believes this to be 'subsidiary' to overgeneration. So we focus here on the account with the most historical precedent-Bonnay ([2], p. 56) writes that it 'might be considered the received view' - and argue that we have not yet been given good reason to go beyond it.

§2. Overgeneration. The overgeneration argument aims to show that the isomorphism invariance test overgenerates by deeming as logical some expressions that intuitively are not. To understand the overgeneration charge, it will be helpful to consider some of the quantifiers that are isomorphism invariant: ${ }^{13}$

$\left(Q_{>\aleph_{0}}\right) Q_{>\aleph_{0}} x \Phi(x)$ is true in a model $\mathcal{M}$ iff uncountably many objects in $\mathcal{M}$ 's domain have the property $\Phi^{\mathcal{M}}$.

$\left(Q_{\aleph_{n}}\right) Q_{\aleph_{n}} x \Phi(x)$ is true in a model $\mathcal{M}$ iff exactly $\aleph_{n}$ objects in $\mathcal{M}$ 's domain have the property $\Phi^{\mathcal{M}}$.

Why is it problematic to judge these quantifiers as logical? The following quote from Bonnay is representative of the worries that these authors have:

this suggests that Tarski's criterion overgenerates and counts too many operations as logical. First, since the aim is to distinguish the realm of logic proper, a proposal which conflates logical notions and mathematical notions does not seem to be on the right track. ([1], p. 37)

\footnotetext{
${ }^{12}$ Very briefly, Feferman [8] proposes replacing bijections with arbitrary surjective functions. One important upshot is that cardinality quantifiers and identity are ruled out as logical. Bonnay [1] endorses invariance under potential isomorphism, which are finite approximations of isomorphisms. As a result, finite quantifiers are counted as logical but higher cardinality quantifiers are not. On both accounts, second- and higher-order quantifiers fail to count as logical, which is how the present overgeneration argument is avoided.

${ }^{13}$ These definitions are shorthand for strict definitions in terms of satisfaction.
} 
The thought is that quantifiers of these sorts are not logical because to call them logical would be to conflate logic and mathematics. On the next page, Bonnay claims that the situation is even worse for isomorphism invariance, since it 'yields a collapse of logic into mathematics' ([1], p. 38). Similarly, Feferman writes that isomorphism invariance 'assimilates logic to mathematics, more specifically to set theory' ([9], p. 3).

The theme in these claims is that isomorphism invariance overgenerates because it somehow involves a confusion of logical and mathematical notions. In particular, it renders certain mathematical notions logical in a way that is problematic.

When we look for cases of overgeneration in these authors' works, the same example always comes up. Feferman writes that, if we accept isomorphism invariance as our criterion of logical nature, 'we can express the Continuum Hypothesis and many other substantial mathematical propositions as logically determinate sentences' ([9], p. 12). Similarly, Bonnay writes that, on the criterion

the quantifier $Q_{\aleph_{1}}$, which tests whether there are exactly $\aleph_{1}$ objects satisfying the formula, is logical. Intuitively, something has gone wrong. Being of size $\aleph_{1}$ is a notion that belongs to set theory, not to logic. ... it is possible to express in $\mathcal{L}_{\infty, \infty}$ the Continuum Hypothesis and other substantial set-theoretic claims. ([1], p. 36)

As is well known, the Continuum Hypothesis is the interpreted first-order sentence which we will abbreviate to:

CH: $2^{\aleph_{0}}=\aleph_{1}$; in words: the cardinality of the power set of the first infinite cardinal is the first uncountable cardinal. ${ }^{14}$

$\mathrm{CH}$ is a controversial, ZFC-undecidable claim. ${ }^{15}$ There are, nevertheless, sentences of second-order logic which are logically true iff $\mathrm{CH}$ is true, and others which are logically true iff $\mathrm{CH}$ is false.

Let $N(X)$ be a second-order formula that is satisfied just if $X$ is equinumerous with the natural numbers, and $R(Y)$ a second-order formula that is satisfied just if $Y$ is equinumerous with the real numbers. These expressions can all be defined in purely second-order vocabulary. ${ }^{16}$ Let $X<Y$ and $X \leq Y$ abbreviate the usual second-order renderings of ' $X$ has a smaller size than $Y$ ' and ' $X$ has a size smaller than or equal to $Y$ ', respectively. Using these abbreviations to keep it manageable and interpreting the quantifiers to range over everything, the interpreted sentence $S$ is a second-order logical truth just when $\mathrm{CH}$ is true:

$$
S: \forall X \forall Y \forall Z((N(X) \wedge R(Y) \wedge X<Z) \rightarrow Y \leq Z)
$$

\footnotetext{
${ }^{14}$ Strictly speaking, $2^{\aleph_{0}}$ is the cardinal equinumerous with the set of functions from $\aleph_{0}$ to $2=\{0,1\}$. Clearly, $\left|\mathbb{P}\left(\aleph_{0}\right)\right|=2^{\aleph_{0}}$.

${ }^{15}$ Thinking of ZFC as a formal theory, we could more strictly write that the first-order sentence in the language of set theory of which $\mathrm{CH}$ is an interpretation is ZFC-undecidable. This reading may be applied to all such claims below.

${ }^{16}$ Shapiro ([23], Section 5.1.2) has the details.
} 
Assuming that $\mathrm{CH}$ is true, the proponent of isomorphism invariance is, therefore, committed to the logical truth of $S$. Of course, the truth of $\mathrm{CH}$ is controversial, but an exactly analogous argument can be run on the assumption that $\mathrm{CH}$ is false with another sentence $S^{\prime}$ that is logically true just if $\mathrm{CH}$ is false.

Defenders of isomorphism invariance as a criterion for logical nature must accept this much. But writers such as Feferman and Bonnay aim to jump from this to a problematic conclusion about $\mathrm{CH}$. Exactly what this problematic conclusion is differs: Feferman claims that $\mathrm{CH}$ is rendered 'logically determinate' ([9], p. 12) by the isomorphism invariance approach and Bonnay claims that it is 'possible to express' ([1], p. 37) $\mathrm{CH}$ using isomorphism-invariant vocabulary.

We will consider five possible arguments that may be intended against isomorphism invariance: that the criterion (i) renders logic sensitive to mathematics in a problematic way; (ii) renders $\mathrm{CH}$ either logically true or false; (iii) renders $\mathrm{CH}$ determinate; (iv) epistemically determines $\mathrm{CH}$; and (v) allows $\mathrm{CH}$ to be logically expressible. We will explain why all five charges should be rejected. Of course, it is open to the critics of isomorphism invariance to put forward another reading, but we have more than covered anything that might be teased out of the existing literature and it is not at all obvious what a plausible alternative reading would look like.

In all of these arguments, it is the status of $\mathrm{CH}$, rather than the isomorphism-invariance test, that is crucial: if you accept the second-order quantifiers as logical for other reasons than isomorphism invariance, then you should still be concerned by the conclusions of these arguments. For this reason, many of our responses have a wider target: they should move not only the opponent of isomorphism invariance but also anyone who believes that the logical status of second-order quantification is problematic. When our arguments in the remainder of this section are directly aimed at the opponent of isomorphism invariance we will be explicit; otherwise, they are aimed at this broader group. The moral about how to distinguish a set-theoretic from a logical claim will emerge later, but can already be exemplified by a brief comparison between $\mathrm{CH}$ and $S$.

$\mathrm{CH}$ is a mathematical sentence, which is not topic-neutral, since it is about sets. Its truth is existentially committing: it requires the existence of the set of functions from $\aleph_{0}$ to 2 , the cardinal $\aleph_{1}$, and of a bijection between them. $\mathrm{CH}$ is therefore not logical, since it is existentially committing and has mathematical content.

$S$, on the other hand, is not a mathematical sentence. It is topic-neutral, since it can be expressed using only logical vocabulary and has no special subject matter. It is not existentially committing: it has the form of a conditional prefaced by three leading universal quantifiers. Finally, $S$ contains only logical expressions. For all of these reasons, $S$ is a logical sentence.

$\mathrm{CH}$ and $S$ are, then, very different sentences and respecting these differences will be crucial in what follows.

2.1. Logic is sensitive to mathematics. The critic of isomorphism invariance believes that the $\mathrm{CH}$ example reveals a certain intimacy between 
mathematics and logic when isomorphism invariance is taken as our criterion of the logical. The thought is that isomorphism invariance renders logical truth sensitive to set theory.

The critic of isomorphism invariance must do more to spell out what 'sensitivity' amounts to in this context. For this reason, we will do much work on our opponents' behalf by providing the four most plausible precisifications of 'sensitivity'. In this section, we work with the intuitive notion of 'sensitivity' that is often appealed to by critics of isomorphism invariance. The discussion in the subsections to follow attempts to give a gloss on this criticism, beginning with a constitutive one in Section 2.2.

Sticking to the rather vague notion of sensitivity, at least a minimal reading must imply that the extension of 'logical truth' varies with the truth value of mathematical sentences: if $\mathrm{CH}$ is true, then $S$ is a logical truth; if $\mathrm{CH}$ is false, then $S^{\prime}$ is a logical truth. This sensitivity to the truth-value of controversial set-theoretic claims, the critic may argue, is reason enough to be suspicious of isomorphism invariance.

To see the mistake here, consider an analogy with an arithmetical case:

A: $2+5=7$

$A^{\prime}:\left(\exists_{2} x F x \wedge \exists_{5} x G x \wedge \forall x \neg(F x \wedge G x)\right) \rightarrow \exists_{7} x(F x \vee G x)$

Here, $A^{\prime}$ is interpreted (as is $A$ of course), ${ }^{17} \exists_{2} x F x$ abbreviates $\exists x \exists y(F x \wedge$ $F y \wedge x \neq y \wedge \forall z(F z \rightarrow(z=x \vee z=y)))$, and likewise for $\exists_{5} x$ and $\exists_{7} x$. $A$ is a truth of arithmetic which is about numbers, hence not topic-neutral nor, by our lights, logical; those who take first-order logic to be logic would agree since $A$ 's first-order formalisation is $f(a, b)=c . A^{\prime}$ is, however, a first-order logical truth. The situation is analogous with the case of $\mathrm{CH}$ : just as $S$ is logically true iff $\mathrm{CH}$ is true, $A^{\prime}$ is logically true iff $A$ is true. The only disanalogy is that the mathematical claim $\mathrm{CH}$ is controversial, whereas everyone accepts the truth of $A$.

The logical truth of $A^{\prime}$ is sensitive to the truth of $A$ in just the same way as the logical truth of $S$ is sensitive to the truth of $\mathrm{CH}$ : again, the extension of 'logical truth' will differ depending on the truth-value of a mathematical claim. The logical truth of both sentences $S$ and $A^{\prime}$ is equivalent to the truth of their corresponding mathematical sentences. And yet this is not thought to be problematic in the first-order case. Now if the sort of sensitivity under discussion is sufficient for overgeneration, then there is overgeneration at the first-order level, which critics of isomorphism invariance typically deny. Therefore, isomorphism invariance does not render the extension of 'logical truth' hostage to mathematics any more than is standard for first-order logic.

One could respond by denying the logical nature of identity, but we will delay discussion of this response until Section 2.6. It should be equally clear from our discussion that isomorphism invariance does not '[require] the existence of set-theoretical entities of a special kind, or at least of their determinate properties' ([9], p. 9). CH (i.e., $2^{\aleph_{0}}=\aleph_{1}$ ) requires the existence

\footnotetext{
${ }^{17}$ In interpreting $A^{\prime}$, we interpret $F$ and $G$ as distinct properties - which ones exactly does not matter.
} 
of sets, namely the set of functions from $\aleph_{0}$ to $2, \aleph_{1}$ and the existence of a bijection between them; similarly for $\neg \mathrm{CH}\left(2^{\aleph_{0}} \neq \aleph_{1}\right)$, minus the existence of the bijection. In contrast, neither $S$ nor $S^{\prime}$ is committed to the existence of a single set, nor a fortiori to any sets having determinate properties.

Another response could be to argue that the arithmetic $A$ and set-theoretic $\mathrm{CH}$ are very different. $A$, the response would go, is much simpler and more familiar than $\mathrm{CH}$, so logic's sensitivity to $A$ is less problematic than to $\mathrm{CH}$. This response is not a straw man: Bonnay ([1], p. 56) distinguishes problematic from unproblematic mathematical content. We believe that this is an unstable distinction. There are two main ways to understand it: that set-theoretic entities are ontologically more problematic than arithmetic ones, or that the former are epistemologically more problematic than the latter. Ontologically, there is no good reason to believe that sets are more problematic than numbers, at least in the present context. On the epistemological side, numbers and sets raise similar access problems. Furthermore, although the truth of $A$ is obvious, there are first-order logical truths whose truth is far from obvious and recognised only on the basis of sophisticated mathematics (Section 2.4 develops an example involving primeness).

Our diagnosis for why someone might think $S$ but not $A^{\prime}$ problematically mathematical is that we tend to be much more familiar with first-order logic. Both $A^{\prime}$ and $S$ are logical truths that have mathematical counterparts, one of which is obviously true and the other far from it, just like $A^{\prime}$ and $S$ respectively. ${ }^{18}$ This verdict will be borne out when we examine more precise versions of the sensitivity argument.

2.2. CH is logically true. We will now consider some precisifications of the thought that logic is sensitive to mathematics on the isomorphism invariance account. In this section, we consider a constitutive reading: $\mathrm{CH}$ is rendered logically true, if true; and logically false, if false. This would be a problematic conclusion for isomorphism invariance, since we motivated it with the thought that logic is topic-neutral and $\mathrm{CH}$ should therefore not count as logical.

There is an interesting parallel here with Etchemendy's arguments against the model-theoretic definition of logical consequence ([7], pp. 123-124). Etchemendy in this passage maintains that the model-theoretic definition is either committed to the claim that $\mathrm{CH}$ is logically true, or the claim that $\mathrm{CH}$ is logically false. As Paseau [19] points out, it is not at all clear how Etchemendy's argument is meant to work here. Paseau shows, further, that no reasonable precisification of Etchemendy's argument is sound. For our purposes, it will be helpful to adapt one of Paseau's precisifications.

$\mathrm{CH}$ is a claim made in (a technical fragment of) natural language, which if fully formalised in first-order terms would be a very long expression in the first-order language of set theory containing a single dyadic predicate $\epsilon$. As noted, it is also existentially committing since for example $2^{\aleph_{0}}=\aleph_{1}$ entails the existence of the cardinals $2, \aleph_{0}$ and $\aleph_{1}$. The sentence that is

\footnotetext{
${ }^{18} \mathrm{On}$ the assumption that $\mathrm{CH}$ is true.
} 
logically true if $\mathrm{CH}$ is true is rather the interpreted second-order sentence $S$, which, recall, is:

$$
S: \forall X \forall Y \forall Z((N(X) \wedge R(Y) \wedge X<Z) \rightarrow(Y \leq Z)) \text {. }
$$

But the logical truth of $S$ is not problematic, assuming $\mathrm{CH}$ is true. Consider again an analogy with an arithmetical case:

$$
\begin{aligned}
A: & 2+5=7 \\
\boldsymbol{A}^{\prime \prime}: & \forall F \forall G\left(\left(\exists_{2} x F x \wedge \exists_{5} x G x \wedge \forall x \neg(F x \wedge G x)\right) \rightarrow \exists_{7} x(F x \vee G x)\right)
\end{aligned}
$$

As with $A^{\prime}, A^{\prime \prime}$ is an interpreted sentence. $A$ is a truth of arithmetic about numbers which is neither topic-neutral nor logical. $A^{\prime \prime}$ is, however, a secondorder logical truth. The situation is as discussed in the previous section: just as $S$ is logically true iff $\mathrm{CH}$ is true, $A^{\prime \prime}$ is logically true iff $A$ is true. If $A$ is true, then the second-order sentence $A^{\prime \prime}$ is logically true. But the logical truth of $A^{\prime \prime}$ is not problematic, since it is topic-neutral. For example, a natural way to express $A^{\prime \prime}$ in English would begin 'for any properties $F$ and $G$, if there are exactly two $F$ s and exactly three $G \mathrm{~s}, \ldots . A^{\prime \prime}$ is therefore a topic-neutral sentence and its logical truth is unproblematic. And similarly for $S$. In each case, the logical sentence (respectively $S$ and $A^{\prime \prime}$ ) can be derived from the mathematical one (respectively $\mathrm{CH}$ and $A$ ) in roughly the following manner: turn the names for cardinals (e.g., the name for $2^{\aleph_{0}}$ in $\mathrm{CH}$ and the name for 2 in $A$ ) into predicate variables whose instances are then specified to have the appropriate numerosity; turn the equal sign into a conditional; universally quantify over the new predicate variables; and, modulo a couple further transformations, one obtains the logical sentence ( $S$ and $A^{\prime \prime}$ respectively). ${ }^{19}$ The crucial point is that neither of the mathematical sentences $\mathrm{CH}$ or $A$ is declared logically true. The fact that they are materially or metaphysically equivalent to logical truths is not enough for them to be logical truths themselves.

To be clear, we are not here assuming that second-order quantifiers are logical expressions. There is a well-known debate about the secondorder quantifiers that is quite independent of isomorphism invariance. For example, if you worry about the logical status of monadic second-order quantification, or its topic-neutrality, you may offer a plural interpretation ([3]). We are not assuming any such justification of the second-order quantifiers. Our argument runs in precisely the opposite direction: isomorphism invariance is motivated by the thoughts that logic is topic-neutral and general. The isomorphism invariance of second-order quantifiers shows that they are topic-neutral and general in the same way as first-order quantifiers. In this way, isomorphism invariance can be used to defend second-order logic as logic (the first of our paper's two subsidiary aims), in a way that dovetails with pluralist justifications of second-order quantification as logical but does not presuppose them. What would be problematic is if an obviously non-topic-neutral sentence like $\mathrm{CH}$ were deemed logically true, if true. And we have shown that it is not.

\footnotetext{
${ }^{19}$ The differences between the two procedures have mainly to do with the fact that the left-hand side of $\mathrm{CH}$ is an exponentiation whereas the left-hand side of $A$ is a sum.
} 
Another way to argue that $\mathrm{CH}$ is rendered logically true on the isomorphism invariance account is to consider the behaviour of the 'it is a logical truth that' operator ${ }^{20}$ If, as we mentioned in Section 1.3, S5 is the correct logic of logical necessity then, if $\mathrm{CH}$ is true, the following is true:

(1) It is a logical truth that $S$.

This, coupled with

(2) $S \leftrightarrow \mathrm{CH}$

is not yet sufficient to entail:

(3) It is a logical truth that $\mathrm{CH}$

since the entailment would involve a modal fallacy. However, we can amend (2) in the following way:

$\left(2^{\prime}\right)$ It is a logical truth that: $S \leftrightarrow \mathrm{CH}$.

(1) and (2') do entail (3).

The problem for our opponents is now twofold. First, they must argue for $\left(2^{\prime}\right)$, which we see no reason to believe. Second, such an argument is not available to our opponents because it proves too much. To return to the arithmetic analogy, $A^{\prime \prime}$ is a logical truth so the analogous conclusion here would be that the arithmetical sentence $A$ is a logical truth. But $A$ is not a logical truth, since it is not topic-neutral. Indeed, to accept $A$ as a logical truth would be to endorse a form of logicism. But logicism is explicitly rejected by at least one of our main opponents (see [8]), and faces numerous well-known objections.

2.3. The indeterminacy of $\mathbf{C H}$. The opponents of isomorphism invariance could put a different spin on the sensitivity objection. They could argue that, on the isomorphism-invariance account, logical determinacy entails a problematic mathematical determinacy. Let $\square_{M}, \diamond_{M}, \square_{L}$ and $\diamond_{L}$ express mathematical necessity, mathematical possiblity, logical necessity and logical possibility respectively, each pair related in the standard way $\left(\square_{M} \equiv \neg \diamond_{M} \neg\right.$ and $\square_{L} \equiv \neg \nabla_{L} \neg$ ).

Our opponents, and Feferman in particular, could argue that logical determinacy leads to a problematic mathematical determinacy as follows. Actually, we present a contraposed version of this reasoning, from mathematical indeterminacy to logical indeterminacy, since we believe it more accurately reflects Feferman's line of thought. First, Feferman [10] holds that $\mathrm{CH}$ is mathematically indeterminate:

(1) $\diamond_{M} C H \wedge \diamond_{M} \neg C H$.

Second, logical truths form a narrower class than mathematical truths. This seems plausible on the isomorphism-invariance view, where logical truths are taken to be completely topic-neutral, whereas mathematical truths may rely on the existence of particular objects. ${ }^{21}$

\footnotetext{
${ }^{20}$ Paseau ([19], p. 46) considers this sort of argument in his response to Etchemendy.

${ }^{21}$ The proponent of isomorphism invariance should reject the converse, since it is once again logicist in flavour and so fits poorly with invariance criteria. We require only the relevant instance of (2) for this argument.
} 
(2) $\square_{L} \phi \rightarrow \square_{M} \phi$.

Third, it is plausible that ' $\mathrm{CH} \leftrightarrow S$ ' is mathematically necessary. It is certainly not logically necessary, as we saw in Section 2.2, since the truth of $\mathrm{CH}$ requires the existence of sets that do not exist of logical necessity, but let's allow that it's mathematically necessary. ${ }^{22}$ Hence:

(3) $\square_{M}(\mathrm{CH} \leftrightarrow S)$

(1)-(3) entail: ${ }^{23}$

(4) $\diamond_{L} S \wedge \diamond_{L} \neg S$

To summarise the objection: if the mathematical truth of $\mathrm{CH}$ is indeterminate, and we accept (2) and (3), which seem plausible, then the logical truth of $S$ is indeterminate. And this may be a commitment that the proponent of isomorphism invariance should not accept.

Our response is, first, that this argument relies on the indeterminacy of $\mathrm{CH}$ as a mathematical truth. If, like us, you believe that the truth of $\mathrm{CH}$ is determinate, then you will reject (1) and will not be troubled by this argument. It should be emphasised that this is the orthodox view. If you subscribe to the orthodox view, then there is nothing here to trouble isomorphism invariance. But if, like Feferman, you believe that the truth of $\mathrm{CH}$ is indeterminate, then we must say more to convince you.

If you hold this view, then you must say more to cash out the notion of mathematical necessity on which the argument crucially depends. One explication of this idea that immediately suggests itself is the set-theoretic multiverse view of e.g., [12]. This view, very roughly, is that there are many different conceptions of set, each of which is instantiated in a corresponding set-theoretic universe. This view provides us with a nice explication of the notion of mathematical necessity, since we can interpret $\square_{M} \phi$ as ' $\phi$ is true in all set-theoretic universes in the multiverse'.

Such multiverse views are, of course, controversial, and we do not accept such a view. But, even if you have some sympathy with the position - or have in mind some other interpretation of $\square_{M}$-we do not believe that this argument should trouble the proponent of isomorphism invariance. This is because, if you accept that the truth of $\mathrm{CH}$ is indeterminate, as you must to think that there's any problem here, and you accept the isomorphism invariance account, then it is very natural to think that the logical truth of $S$ is likewise indeterminate. This is because isomorphism invariance seeks to complement the model-theoretic definition of logical consequence, which is a set-theoretic definition. If you accept isomorphism invariance, therefore, you should accept a model-theoretic definition of logical truth, i.e., a sentence is a logical truth just if it is true in all domains under all reinterpretations of the invariant vocabulary. But if you believe that there are indeterminate sentences about the background universe of sets, then it is unsurprising that

\footnotetext{
${ }^{22}$ If you doubt that ' $\mathrm{CH} \leftrightarrow S$ ' is mathematically necessary, then this version of the overgeneration argument doesn't get off the ground. for $\square_{M}$.
} 
some of this indeterminacy carries over to logical truth. This is not to say that any mathematical sentence is logically true, for reasons given earlier in this section. The point, rather, is that if you hold that $\mathrm{CH}$ is indeterminate, then you should not be troubled, or even surprised, that there are some sentences whose logical status is indeterminate. The hybrid position that sees the mathematical sentence $\mathrm{CH}$ as indeterminate and the logical truth of the second-order sentence $S$ as determinate seems ill-motivated.

In sum, the thought that the truth of $\mathrm{CH}$ is mathematically indeterminate is highly controversial. If you reject it, then you will not think there is anything here to threaten isomorphism invariance. If you do have sympathy with that controversial view, however, then we have argued that your view is fully compatible with isomorphism invariance. The link from logical determinacy to mathematical determinacy has not been shown to be problematic.

2.4. CH is epistemically determined. We now turn to our next reading of sensitivity: $\mathrm{CH}$ is epistemically determined by isomorphism invariance. The thought here is that logic alone should not force us to accept or deny controversial claims of set theory, so isomorphism invariance is not the correct criterion for logical nature.

First, it will be instructive to rehearse the argument of Section 2.2, but with the operator 'it is an a priori truth that' in place of 'It is a logical truth that':

(1) It is an a priori truth that $S$.

This, coupled with

(2) It is an a priori truth that: $S \leftrightarrow \mathrm{CH}$. entails

(3) It is an a priori truth that $\mathrm{CH}$.

This argument, unlike its analogue for logical truth, is sound (assuming Distribution for 'it is an priori truth that'). But this sort of conclusion should not worry us, or at least it should not worry anyone inclined to think that $S$ is knowable a priori: if $\mathrm{CH}$ is true then presumably it is knowable a priori. What should worry us is the logical truth of $\mathrm{CH}$ and we have not seen any sound argument for that conclusion.

Returning to the criticism that $\mathrm{CH}$ is epistemically determined by isomorphism invariance, we accept, of course, that $\mathrm{CH}$ is an unsettled claim of set theory. What we deny is the conditional claim that if isomorphism invariance is the correct criterion for logical nature, then some new and problematic epistemic route to knowledge of $\mathrm{CH}$ or of $\neg \mathrm{CH}$, as the case may be, becomes available.

To begin with, let us be clear about the expected order of discovery. The criticism seems to imply that we might first discover that $S$ is a logical truth and thereby infer the truth of $\mathrm{CH}$. But there is another sentence $S^{\prime}$, which is logically true just if $\mathrm{CH}$ is false, ${ }^{24}$ and $S^{\prime}$ also contains only isomorphisminvariant vocabulary. So isomorphism invariance as a criterion for logical

\footnotetext{
${ }^{24}$ See once more Shapiro ([23], Section 5.1.2) for details of how to construct $S^{\prime}$.
} 
nature is consistent with both the truth of $\mathrm{CH}$ (in which case $S$ is logically true and $S^{\prime}$ is false) and the falsity of $\mathrm{CH}$ (in which case $S^{\prime}$ is logically true and $S$ is false). In short, it is not isomorphism invariance alone that delivers the logical truth of $S$ : it is isomorphism invariance and the truth-value of $\mathrm{CH}$.

If we ever settle CH's truth-value, the expected order of discovery is rather that we shall first determine the truth of $\mathrm{CH}$ via set-theoretic techniquesfrom mathematically well-motivated extensions of $\mathrm{ZFC}$ - and then infer the logical truth of $S$ (and similarly for $S^{\prime}$ ). The proponent of isomorphism invariance is in no way committed to, indeed rejects, the idea that 'a competent speaker should be able, on the basis of his or her semantic competence, to accept or reject the Continuum Hypothesis'. ${ }^{25}$ Even in the first-order case, in which there is a sound and complete proof procedure, the order of discovery may very well run from mathematics to logic. For example, there is a statement $\phi_{N}$ of first-order logic materially equivalent to the arithmetical statement ' $N$ is prime'. ${ }^{26}$ Advances in say analytic number theory may determine that a particular number $N$ is prime, thereby yielding knowledge of the logical truth $\phi_{N} \cdot{ }^{27}$

Second, exploiting logical knowledge to yield mathematical knowledge should not be regarded as problematic. Again, this should be familiar enough from the first-order case, e.g., our ' $N$ is prime' example. From knowledge of the material biconditional ' $N$ is prime iff $\phi_{N}$ is true' and logical knowledge of the fact that $\phi_{N}$ is true (say via a premiss-free first-order proof), one may come to know that $N$ is prime. Coming to know that $\mathrm{CH}$ is true analogously, via knowledge of $S$ and the biconditional $S \leftrightarrow \mathrm{CH}$, is no more problematic than coming to know that $N$ is prime by exploiting knowledge of the logical truth $\phi_{N}$. As mentioned in the previous paragraph, the converse is also true: we can and do exploit mathematical knowledge to yield knowledge of logical truth.

Third, and related to the second point: that some logical knowledge has been exploited to yield mathematical knowledge does not mean that only logical knowledge has been exploited. In our arithmetical example, logical knowledge of the primality of $N$ only follows from logical knowledge of $\phi_{N}$ and logical knowledge of the material biconditional ' $N$ is prime iff $\phi_{N}$ is true'. Suppose, as we believe, that the biconditional is not logically known. Then this route to knowledge of the primality of $N$ is not purely logical, as it rests on extra-logical knowledge of the biconditional. Similarly, coming

\footnotetext{
${ }^{25}$ Bonnay ([1], p. 65), with the original 'its' replaced by 'his or her'.

${ }^{26}$ The statement $\phi_{N}$ is the negation of $\bigvee_{\substack{1<b<N \\ 1<a<N}} \operatorname{Mult}(a, b, N)$, a disjunction which consists of $(N-2)^{2}$ disjuncts. Mult $(a, b, N)$ is the first-order formalisation of the following statement: If there are exactly $a F \mathrm{~s}$, and each $F$ is $R$-related to exactly $b G \mathrm{~s}$, and no two distinct $F \mathrm{~s}$ are ever $R$-related to the same $G$, and any $G$ is the $R$-relatum of some $F$, then there are exactly $N G$ s.

${ }^{27}$ Analytic number theory considers the natural numbers as embedded in the complex plane and uses techniques from complex analysis to derive facts about them, in particular facts about primes. The canonical proof of the Prime Number Theorem is a famous case in point.
} 
to know $\mathrm{CH}$ via logical knowledge of $S$ and knowledge of the biconditional ' $\mathrm{CH}$ iff $S$ ' yields logical knowledge of $\mathrm{CH}$ only on the assumption that the biconditional is logically known. Yet we see no reason on the isomorphism invariance account to suppose that this biconditional is logically knowable.

In sum, knowledge of $S$ may in principle lead to knowledge of $\mathrm{CH}$, although the order of discovery is almost bound to run in the other direction. As for logical knowledge of S supposedly leading to logical knowledge of $\mathrm{CH}$, not only has the case for this supposition not been made, it also seems highly problematic.

2.5. CH is logically expressible. The last three subsections can be seen as making more precise in different ways the criticism that isomorphism invariance renders logic sensitive to mathematics. We turn finally and more briefly to Bonnay's claim that it is possible to express $\mathrm{CH}$ and other complex settheoretic claims using only isomorphism-invariant expressions ([1], p. 38). We can now see that this objection is misguided: what is expressible in purely logical vocabulary is not $\mathrm{CH}$ but $S$. And if, as we have argued, the logical truth of $S$ is unproblematic, then of course its expressibility is unproblematic, since a sentence's logical truth (in a particular logic) implies its expressibility (in that logic). If a sentence is a logical truth of second-order logic, then of course it can be expressed in the language of second-order logic. And if the former should not trouble us, nor should the latter.

Now mathematicians typically do not make fine distinctions between statements expressing what they might regard as the same 'mathematical content'. Thus they might well uncritically say that $A$ and $A^{\prime}$ or $A^{\prime \prime}$ express the same thought, and that the same goes for the sentences $S$, $Q_{2 \aleph_{0}} x(x=x) \leftrightarrow Q_{\aleph_{1}} x(x=x)$ and $\mathrm{CH}$. But when our interest is in the precise demarcation between mathematics and logic, it is important to tread carefully. The sentence $A$ is an arithmetical truth, because its formalisation $f(a, b)=c$ is not valid on all models. The sentence $A^{\prime \prime}$ in contrast, or for that matter its first-order version $A^{\prime}$ obtained by dropping the two leading second-order quantifiers, is logically true. Similarly, $\mathrm{CH}$ is not a logical truth, since it is existentially committing; indeed, it turns out to not be provable in first-order ZFC, never mind first-order logic without nonlogical axioms. In contrast, $S$ is a logical truth. Responding to this or closely related objections, philosophers have been wont to concede that there is no boundary between logic and mathematics (e.g., [24], p. 146). But-and this is our third moral that concession would be premature. According to isomorphism invariance, there is a principled boundary between logic and mathematics: sentences such as $S$ lie on one side of it, whereas sentences such as $\mathrm{CH}$ lie on the other.

2.6. Identity. At this point, there is a possible objection that we must address. Our responses to the critics of isomorphism invariance have all involved relations between simple arithmetic claims and first-order logical truths. But these logical truths all involve numerically definite quantification and so rely crucially on the logical nature of identity.

Our reply is twofold. First, the logical status of identity is widely accepted and largely uncontentious; several theoretical accounts of logical 
constanthood endorse it as logical, including not just isomorphism invariance but also inferentialist accounts such as [21] and [17]; and the arguments against identity being logical are thin on the ground and unpersuasive. Nevertheless, we recognise that in this context our first reply is dialectically controversial. In particular, Feferman - one of the main proponents of the overgeneration objection - denies the logical nature of identity and puts forward a homomorphism invariance criterion which rules identity out as logical. ${ }^{28}$

Our second reply is therefore that although our simple arithmetical examples have so far involved the logical nature of identity, other examples are available that do not rely on this assumption. Consider the following pair of sentences:

$$
\begin{aligned}
\boldsymbol{G}: & 3 \geq 2 \\
\boldsymbol{G}^{\prime}: & \exists x \exists y \exists z(F x \wedge \neg F y \wedge G y \wedge \neg G z \wedge H x \wedge \neg H z) \rightarrow \exists x \exists y(F x \wedge \neg F y) .
\end{aligned}
$$

If the mathematical sentence $G$ is true, then the interpreted sentence $G^{\prime}$ is logically true. The situation is analogous to $\mathrm{CH}$ and $S$ but, crucially, $G^{\prime}$ does not rely on the logical nature of identity: $G^{\prime}$ only includes vocabulary that Feferman would accept as logical. So, if you are at all unsure about the logical nature of identity, then you can recast the arguments of Sections 2.1-2.2 in terms of $G$ and $G^{\prime}$, rather than $A$ and $A^{\prime}$ or $A^{\prime \prime}$.

2.7. Logical notions. We have argued that no version of the overgeneration argument against isomorphism invariance is convincing. We end our discussion of the overgeneration argument by considering a final, related worry voiced by a critic of isomorphism invariance. As Bonnay ([1], pp. 36-37) sees it, the overgeneration argument is not limited to statements. The charge is that isomorphism invariance classifies certain notions that are in fact mathematical as logical. We have focussed on the logical truth of various statements but, this objection goes, that is of secondary concern: the real worry is that a notion such as 'there exists uncountably many' has been deemed logical, when intuitively it shouldn't.

We respond to the general version of this objection first, before considering a particular topological example offered by Bonnay. An important theme of this paper has been the need to keep various closely related statements separate. It it tempting to say, for example, that $S$ and $\mathrm{CH}$ express the same content, or similar. We have argued that this way of speaking is, strictly speaking, incorrect and that, when we avoid it, overgeneration worries dissolve. Our reply to this objection is similar: just as it is important not to conflate various statements, it is important not to conflate various notions.

The objection that some notion ought or ought not to be classified as logical is we believe much harder to assess than the objection that some statement (such as $\mathrm{CH}$ ) ought or ought not to be classified as logical. This is natural, since we have clearer (though still messy) intuitions about the extension of 'logical truth' than 'logical constant'. It would be very difficult,

\footnotetext{
${ }^{28}$ By Feferman's criterion, the first-order quantifiers are logical but not identity, cardinality quantifiers or second-order quantifiers.
} 
for example, to introduce a novice to the concept of logical constants before explaining the concept of logical truth and consequence. Although there is no space to develop this line of thought here, we believe that any plausible attempt to draw a principled line between logical and mathematical notions will have to rely at least in part on judgments about various statements' logicality. In as much as we do have a pretheoretic grip on whether a notion, as opposed to a statement, is logical or not, it is not at all clear that we - meaning mathematicians and the mathematically well-educated - tend to think of general mathematical notions as nonlogical. The notion of a topological space, or of a continuous function between two topological spaces, or of a group, or of a homomorphism between two groups, and so on, are taken to be maximally general notions whose instances are anything whatsoever that satisfies the defining conditions. Naturally, the study of such notions belongs to mathematics. But the notions themselves - as opposed to claims that something instantiates them - are entirely general and apply to anything that satisfies some structuring conditions, as David Hilbert was one of the first to note.

What is true is that one can perfectly well express $\mathrm{CH}$, to within a reasonable informal standard of mathematical equivalence, by using various generalised quantifiers. We have suggested that this does not commit one to set-theoretic objects (e.g., infinite cardinals) because these quantifiers are isomorphism-invariant primitives. But are not ontological commitments concealed by such a move? What does it mean to say that the quantifier 'there are continuum many' does not carry set-theoretic commitments if we can justify or even understand assertions involving it only by relating the subject matter by a set-theoretic isomorphism to a canonical example of continuum size?

Our retort to such an objection is threefold. First, as noted in Section 2.4, it is a fact that mathematics can be used to yield knowledge of logical truths; as mentioned, this applies to first- as well as second-order logical truths. And as noted in Section 2.1, there is no relevant disanalogy between cardinality quantifiers expressible in first-order terms such as $\exists_{512}$ on the one hand and higher-order ones such as $Q_{>\aleph_{0}}$ on the other. Arithmetic is no less part of mathematics than set theory, the familiarity of first-order logic notwithstanding. Our contention is that both these quantifiers are logical, and we see no principled reason to regard the latter as 'mathematical' or 'problematically mathematical' but the former not. Second, we deny that understanding an assertion implies an ontological commitment to the entities over which it quantifies. For example, we can perfectly well understand discourse about fictional objects (e.g., dragons or fairies), or metaphorical language, without thereby incurring commitment to the objects described.

Finally, the use of set theory to grasp a sentence such as $S$ is only a crutch, though admittedly a very helpful one. The sentence $S$ can in principle be understood literally, using only the conceptual resources expressed by a second-order language free of nonlogical terms and without deploying mathematical concepts. In much the same way, the statement $A^{\prime}$ in Section 2.1 
can be understood by deploying the conceptual resources of first-order logic, without ever entertaining the thought that $2+5=7$. We realise, of course, that it is very hard for limited human cognisers to understand $S$ without using set theory as a crutch. It would be just as hard for us to understand the $10^{10^{10}}$-termed Boolean connective $*_{\text {Fermat }}$, which outputs the truth-value True iff the number of True inputs equals $N$ for $N$ the index in a true Fermat equation, ${ }^{29}$ without recourse to number theory. But we take it that the philosophically interesting notion of conceptual dependence at stake here is an in-principle one that abstracts from contingencies of human implementation rather than an in-practice notion that depends on the specific mechanisms of human cognition.

We turn finally to Bonnay's example of a continuous function between topological spaces, intended to make his objection sharper. Briefly stated, the notion

$\left(X, \tau_{X}\right)$ and $\left(Y, \tau_{Y}\right)$ are topological spaces and $f:\left(X, \tau_{X}\right) \rightarrow\left(Y, \tau_{Y}\right)$ is continuous

may be seen to be isomorphism-invariant when one unpacks the definitions (here $\tau_{X}$ and $\tau_{Y}$ are the topologies on $X$ and $Y$ respectively). The reason is that for $f:\left(X, \tau_{X}\right) \rightarrow\left(Y, \tau_{Y}\right)$ to be continuous, it matters not what the elements of $X$ or $Y$ are, but only that the preimage under $f$ of any element of $\tau_{Y}$ is an element of $\tau_{X}$, a fact that is preserved under isomorphism. ${ }^{30}$ More generally, any class of structures closed under isomorphism gives rise to a logical notion that applies to all and only elements of that class.

Two replies may be made to this challenge. First, it seems to be an inherent feature of the invariantist account. The invariantist carves up the space of models into equivalence classes, identifying logical notions as (all and only) those that respect the equivalence class structure (i.e., they apply in some way to a particular model iff they apply in the same way to all its equivalents, for the given notion of equivalence). To illustrate the point briefly, consider Bonnay's own proposal that the correct equivalence relation is not isomorphism but potential isomorphism. ${ }^{31}$ This account makes the notion 'structure potentially isomorphic to $\langle\mathbb{R},<\rangle$ ' logical; in this case, the class includes not just structures isomorphic to $\langle\mathbb{R},<\rangle$ but also structures such as $\langle\mathbb{Q},<\rangle$.

\footnotetext{
${ }^{29} N$ is an index in such an equation iff there are positive integers $x, y, z$ such that $x^{N}+y^{N}=z^{N}$. Andrew Wiles' proof shows that $*_{\text {Fermat }}$ outputs True iff exactly 0,1 or 2 of the inputs are True.

${ }^{30}$ Meaning that if the topological spaces $\left(X, \tau_{X}\right)$ and $\left(X^{\prime}, \tau_{X^{\prime}}\right)$ are isomorphic-i.e., homeomorphic - via the bijection $i_{X X^{\prime}}: X \rightarrow X^{\prime}$, and $\left(Y, \tau_{Y}\right)$ and $\left(Y^{\prime}, \tau_{Y^{\prime}}\right)$ are homeomorphic via the bijection $i_{Y Y^{\prime}}: Y \rightarrow Y^{\prime}$, then $f:\left(X, \tau_{X}\right) \rightarrow\left(Y, \tau_{Y}\right)$ is continuous iff $f^{\prime}:\left(X^{\prime}, \tau_{X^{\prime}}\right) \rightarrow\left(Y^{\prime}, \tau_{Y^{\prime}}\right)$ is continuous, where $f^{\prime}=i_{Y Y^{\prime}} \circ f \circ i_{X X^{\prime}}^{-1}$.

${ }^{31} \mathrm{~A}$ potential isomorphism $I$ between two structures $\mathcal{M}$ and $\mathcal{M}^{\prime}$ is a nonempty set of partial isomorphisms such that for all $f \in I$ and $a$ in the domain of $\mathcal{M}$ (respectively $b$ in the domain of $\mathcal{M}^{\prime}$ ) there is a $g \in I$ with $f \subseteq g$ and $a \in \operatorname{dom}(g)$ (respectively $b \in \operatorname{ran}(g)$ ). A partial isomorphism $f \in I$ between $\mathcal{M}$ and $\mathcal{M}^{\prime}$ is an isomorphism between a substructure of $\mathcal{M}$ and a substructure of $\mathcal{M}^{\prime}$.
} 
Second and relatedly, determining whether or not some function between topological spaces is continuous or not seems to be a matter of applying definitions. In this intuitive sense at least, it is a logical notion. Take for example the topological spaces $X$ and $Y$ and function $f$ between them given by:

$$
\begin{aligned}
& X=\{a, b\} \text { and } \tau_{X}=\{\emptyset, X,\{a\},\{b\}\} \\
& Y=\{c, d\} \text { and } \tau_{Y}=\{\emptyset, Y,\{c\}\} \\
& f(a)=c ; f(b)=d
\end{aligned}
$$

As a matter of first-order logic, the conclusion that $f$ is continuous follows from these conditions. More precisely, consider the conditional whose antecedent is the conjunction of the above facts about $\left(X, \tau_{X}\right),\left(Y, \tau_{Y}\right)$ and $f$ in appropriately unpacked set-theoretic notation (so that for example $X=\{a, b\}$ becomes $\forall z(z \in X \leftrightarrow z=a \vee z=b))$, and whose consequent states that $f$ is continuous, i.e., that the preimage under $f$ of every element of $\tau_{Y}$ is an element of $\tau_{X}$. Availing ourselves of the usual quantifier restriction conventions, the conditional's consequent is the statement

$$
\begin{aligned}
& \forall O_{Y} \in \tau_{y} \exists O_{X} \in \tau_{X}\left(\forall x \in O_{X}\left(f(x) \in O_{Y}\right) \wedge \forall y \in O_{Y} \exists x \in\right. \\
& \left.O_{X}(y=f(x))\right)
\end{aligned}
$$

The resulting conditional is a first-order validity. We may also formalise the statement of $f$ 's continuity in a higher-order logic. ${ }^{32}$ Either way, no extralogical, somehow purely mathematical, premiss is needed to determine $f$ 's continuity.

In sum, if the type of facts required to classify any given function from one topological space to another as continuous are what determine whether the notion of a continuous function is logical or not, then this notion is indeed logical since nothing but logic is required for the classification. Of course, in contrast to the simple examples $\left(X, \tau_{X}\right)$ and $\left(Y, \tau_{Y}\right)$ above, some topological spaces are not first-order definable, so the notion of a continuous function between such spaces will not admit first-order characterisation. But what is not first-order expressible in these cases is the description of the spaces themselves or the description of the function in question, not the fact that the function is continuous, which remains the (first-order) consequent of the conditional, as specified above. Contrast this with an empirical notion, say that of a car: to determine whether some specified object is a car one needs empirical information about that object. The same point generalises to other mathematical structures and maps between them (groups and group homomorphisms and so on).

§3. Conclusion. A demarcation of the logical constants is crucial in order to supplement the orthodox account of logical consequence. We have defended an isomorphism-invariance approach to this demarcation. We began with the vague worry that logic may have been rendered sensitive to mathematics if we accept this demarcation. The worry has been shown

\footnotetext{
${ }^{32}$ So that $\tau_{X}$ and $\tau_{Y}$ become second-order predicate constants. The consequent becomes $\forall F\left(\tau_{Y} F \rightarrow \exists G\left(\tau_{X} G \wedge \forall x(G x \rightarrow F f(x)) \wedge \forall y(F y \rightarrow \exists x(y=f(x) \wedge G x))\right)\right)$.
} 
to be illusory, however: there is no good sense of 'sensitive' relating logic to mathematics in a problematic way. We have worked on our opponents' behalf to spell out the notion of 'sensitivity' in play. Many of the arguments we have put forward can also be used to defend second-order quantification as logic. And we have also noted that the boundary between mathematics and logic is firmer than is often thought. We have not established the correctness of isomorphism invariance: the issues of undergeneration and of the account's purely extensional nature remain. But the former has not been developed in any great detail and a purely extensional account is sufficient for many purposes. Isomorphism invariance may yet turn out to be false, but it is not false for the reason usually given. It remains an attractive account of logical nature.

§4. Acknowledgments. We would like to thank Patricia Blanchette, Denis Bonnay, Tim Button, Solomon Feferman, Salvatore Florio, Gila Sher and an anonymous referee for their helpful comments on previous drafts. We would also like to thank audiences in Cambridge, Munich and Oxford.

\section{REFERENCES}

[1] D. Bonnay, Logicality and invariance, this BULletin, vol. 14 (2008), pp. 29-68.

[2] - Logical constants, or how to use invariance in order to complete the explication of logical consequence. Philosophy Compass, vol. 9 (2014), pp. 54-65.

[3] G. Boolos, Nominalist platonism. Philosophical Review, vol. 94 (1985), pp. 327-344.

[4] J. P. Burgess, Which modal logic is the right one? Notre Dame Journal of Formal Logic, vol. 40 (1999), pp. 81-93.

[5] E. CASANOvas, Logical operations and invariance. Journal of Philosophical Logic, vol. 36 (2007), pp. 33-60.

[6] C. Dutilh Novaes, The undergeneration of permutation invariance as a criterion for logicality. Erkenntnis, vol. 79 (2014), pp. 81-97.

[7] J. Etchemendy, The Concept of Logical Consequence, Harvard University Press, Cambridge, MA, 1990.

[8] S. Feferman, Logic, logics and logicism. Notre Dame Journal of Formal Logic, vol. 40 (1999), pp. 31-54.

[9] - Set-theoretical invariance criteria for logicality. Notre Dame Journal of Formal Logic, vol. 51 (2010), pp. 3-20.

[10] - Is the Continuum Hypothesis a definite mathematical problem? unpublished manuscript, 2011.

[11] - Which quantifiers are logical? A combined semantical and inferential criterion, Quantifiers, Quantifiers, and Quantifiers: Themes in Logic, Metaphysics and Language (A. Torza, editor), Springer, 2015, pp. 19-30.

[12] J. D. Hamkins, The set-theoretic multiverse. Review of Symbolic Logic, vol. 5 (2012), pp. 416-449.

[13] J. MACFARLANE, What does it mean to say that logic is formal? Ph.D. dissertation, University of Pittsburgh, 2000.

[14] - Logical constants, The Stanford Encyclopedia of Philosophy (Fall 2015 edition) (E. Zalta, editor), 2015.

[15] T. McCARTHY, The idea of a logical constant. Journal of Philosophy, vol. 78 (1981), pp. 499-523.

[16] V. McGee, Logical operations. Journal of Philosophical Logic, vol. 25 (1996), pp. 567-580. 
[17] P. MiLne, Existence, freedom, identity, and the logic of abstractionist realism. Mind, vol. 116 (2007), pp. 23-53.

[18] A. Oliver, The matter of form: Logic's beginnings, The Force of Argument: Essays in Honor of Timothy Smiley (A. Oliver and J. Lear, editors), Routledge, London, 2010, pp. 165-185.

[19] A. C. Paseau, The overgeneration argument (s): A succinct refutation. Analysis, vol. 74 (2014), pp. 40-47.

[20] C. Peacocke, What is a logical constant? Journal of Philosophy, vol. 73 (1976), pp. 221-240.

[21] S. READ, Identity and harmony. Analysis, vol. 64 (2004), pp. 113-119.

[22] G. SAGI, The modal and epistemic arguments against the invariance criterion for logical terms. Journal of Philosophy, vol. 112 (2015), pp. 159-167.

[23] S. ShAPIRO, Foundations without Foundationalism, Oxford University Press, Oxford, 1991.

[24] - Logical consequence: Models and modality, The Philosophy of Mathematics Today (M. Schirn, editor), Oxford University Press, New York, 1998, pp. 131-156.

[25] G. SHER, The Bounds of Logic, MIT Press, Cambridge, MA, 1991.

[26] — , The foundational problem of logic, this BulLETIN, vol. 19 (2013), pp. 145-198.

[27] - Epistemic Friction, Oxford University Press, Oxford, 2016.

[28] A. Tarski, O pojciu wynikania logicznego. Przeglad Filozoficzny, vol. 39 (1963), pp. 58-68, transl. by J. Woodger as 'On the concept of logical consequence' and repr. in Tarski's Logic, Semantics, Metamathematics, second ed. (J. Woodger, editor), Hackett, Indianapolis, pp. 409-420.

[29] - Introduction to Logic and the Methodology of the Deductive Sciences, Dover, New York, 1937. Refs to 1995 reprint.

[30] - What are logical notions? History and Philosophy of Logic, vol. 7 (1966), pp. 143-154. Refs to 1986 reprint.

[31] L. H. Tharp, Which logic is the right logic? Synthese, vol. 31 (1975), pp. 1-21.

[32] J. Woods, Logical indefinites. Logique et Analyse, vol. 57 (2014), pp. 277-307.

\author{
ST JOHN'S COLLEGE \\ UNIVERSITY OF CAMBRIDGE \\ CAMBRIDGE CB2 1TP, UK \\ E-mail: oeg21@cam.ac.uk \\ WADHAM COLLEGE \\ UNIVERSITY OF OXFORD \\ OXFORD OX1 3PN, UK \\ E-mail: alexander.paseau@philosophy.ox.ac.uk
}

